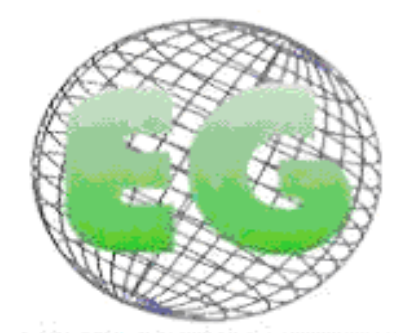

ISSN 1695-6141 N 27

www.um.es/egloball

\title{
REVISIONES
}

\section{Rol de enfermería en la prevención del delirium en ancianos hospitalizados con fractura de cadera. Recomendaciones generales.}

\author{
The role of nursery in the prevention of the delirium in elderly hospitalized with hip fracture. General \\ recommendations.
}

\section{${ }^{*}$ Carrera Castro, Carmen.}

\begin{abstract}
*DUE. Servicio Hospitalario de Cirugía Ortopédica Traumatológica (COT). Hospital Ernest LLuch. Calatayud. Zaragoza.
\end{abstract}

Palabras clave: delirium; fractura de cadera; factores de riesgo; prevención; ancianos. Keywords: Delirium; Hip fracture; Risk factors; Prevention; Elderly.

\section{RESUMEN}

Se realiza una revisión bibliográfica de la literatura en las principales bases biomédicas para identificar cuáles son los factores de riesgo modificables o controlables en ancianos hospitalizados con fractura de cadera, susceptible de padecer delirium, y crear un Plan de Actuación de Recomendaciones de Enfermería desde el ingreso hasta el alta en la unidad, basándonos en la prevención, con el propósito de fomentar el descenso en la frecuencia y consecuencias desfavorables del delirium.

Son pocos y con gran disparidad numérica en los resultados obtenidos entre los diferentes trabajos que estudian la eficacia de adoptar medidas ambientales como estrategias en la prevención del delirium. Las acciones llevadas a fin, en as de la prevención, son primordialmente las que controlan los factores de riesgo como: el deterioro cognitivo y físico, inmovilidad, discapacidad visual y auditiva, deshidratación, desnutrición, desorientación, privación del sueño, polifarmacia y dolor.

Al delirum no se le presta la importancia que requiere, a pesar de su alta frecuencia e impacto bio-psico-socioeconómico en el paciente y su entorno. La prevención es la medida más eficaz y económica de abordar el delirium en su fase inicial y la enfermería contribuye en gran medida en esta labor. El anciano hospitalizado con fractura de cadera es un paciente de alto riesgo debido al gran número de factores de riego agregados a su situación clínica y contexto.

Sería interesante en otras investigaciones futuras valorar la eficacia de las recomendaciones ofrecidas en esta revisión.

\section{ABSTRACT}

A bibliographical revision of Literature is done in the main biomedical databases to identify which are the modifiable or controllable risk factors in elderly hospitalized with hip fracture, susceptible to suffer delirium, and to create a Plan of Action of Recommendations of Infirmary from the admission to the discharge in the unit, 
basing ourselves on the prevention, in order to promote the reduction in the frequency and unfavourable consequences of the delirium.

They are few and with great numerical disparity the obtained results between the different works that study the effectiveness to adopt environmental measures like strategies in the prevention of the delirium. The taken actions to aim, in ace of the prevention, are fundamentally those that control the risk factors such as: the mental and physical deterioration, immobility, visual and auditory disability, dehydration, undernourishment, disorientation, deprivation of the dream, polypharmacy and pain.

The delirium is not given the importance that requires in spite of its high frequency and the biological, psychological, social and economic impact in the patient and his/her environment. The prevention is the most effective and economic measure to approach the delirium in his/her starting phase and the nursery contributes to a great extent in this work. The elderly hospitalized with hip fracture are patients of high risk due to the great number of risk factors added to its clinical situation and context.

It would be interesting in other future investigations to value the effectiveness of the recommendations offered in this revision.

\section{INTRODUCCIÓN}

El Síndrome Confusional Agudo (SCA) o Delirium a lo largo de los tiempos se le ha ido denominando con una gran pluralidad de términos, el "delirium" el más utilizado y aceptado por consenso internacional. ${ }^{1-2}$

Es un trastorno psiquiátrico severo frecuente ${ }^{3-7}$ con rangos de incidencias dispares dependiendo de la población clínica a estudio, método de detección y criterios diagnósticos utilizados $^{8}$, siendo los ancianos hospitalizados con fractura de cadera los que tienen una frecuencia mayor con respecto a otros grupos de estudio ${ }^{9-13}$, la incidencia aumenta con la edad, se dan más en ancianos ${ }^{3,4,14}$. Es el segundo síndrome psiquiátrico más prevalente después de los trastornos depresivos en la esfera hospitalaria ${ }^{15}$ y la cuarta complicación más tratada en pacientes hospitalizados con fractura de cadera ${ }^{16}$.

El delirium no solo es importante por la alta frecuencia que tiene sino por el impacto negativo de sus consecuencias (Tabla IV). Un inadecuado abordaje de este síndrome puede repercutir nocivamente en el pronóstico y evolución del paciente.

Su etiología es multifactorial ${ }^{4,7,13}$ con base orgánica ${ }^{17,18}$, es prevenible ${ }^{19,20}$ y reversible ${ }^{17,18,21,22}$ de inicio agudo, transitorio y curso fluctuante en horas y días, con alteración del ciclo vigilia-sueño, se manifiesta por una alteración de la conciencia y cambios de las funciones cognoscitivas ${ }^{23}$. Puede presentarse desde un punto de vista clínico de forma hiperactiva (agitación, inquietud), hipoactivo (somnolencia, aletargado) y mixta (combinación de los anteriores) ${ }^{24}$.

El objetivo de esta revisión es centrarnos en la prevención (primaria y secundaria) del delirium, a través de una Plan de recomendaciones durante la estancia hospitalaria en una unidad de traumatología, desde la perspectiva de enfermería, mediante la disminución o eliminación de los factores de riesgo modificables con mayor implicación en su aparición, y la detección precoz de su sintomatología, en pacientes ancianos con fractura de cadera, por ser un grupo de riesgo altamente vulnerable (edad avanzada, encamado, hospitalizado, con dolor, fracturado, cirugía ortopédica...) con elevada incidencia y prevalencia de padecerlo, en colaboración con un equipo sanitario multidisciplinario y la familia (un apoyo importante en este proceso $)^{25}$. 
La prevención primaria sería la estrategia pilar más efectiva a la hora de abordar en sus inicios al delirium porque una vez que brota, las medidas de intervención son menos eficientes y eficaces ${ }^{25-27}$, al igual que con una rápida identificación y adhesión de las acciones terapéuticas adecuadas (prevención secundaria) para minimizar los efectos del delirium se evita mayores complicaciones médicas en estos pacientes frágiles y vulnerables $^{4,28}$.

Prevenir el delirium no es más que mantener un equilibrio homeostático del celebro y limitar los agentes estresantes del medio ambiente que puedan afectar negativamente al anciano hospitalizado. El contacto continuado y estrecho de la enfermería con el paciente y la familia juega un papel primordial en las estrategias de prevención y en todo el proceso de la hospitalización.

\section{Diagnóstico. DSM-IV}

Su diagnostico se debe considerar como una urgencia médica y es básicamente clínico (historia clínica (factores médicos y sociales), entrevistas con familiares o cuidadores, exploración fisca general, exámenes psicológicos y pruebas complementarios).

Un diagnóstico precoz del cuadro, de su etiología y de los factores de riesgo permite prevenir sus consecuencias adversas y complicaciones derivadas de su falta de tratamiento $^{29}$, siendo la más trágica el éxitus (Tabla IV).

El criterio diagnóstico según el Manual Diagnóstico y Estadístico de Trastornos Mentales (DSM-IV-TR) ${ }^{23}$ lo define como: Tabla I.

\section{TABLA I Criterios Diagnósticos del Delirium. DSM-IV-TR.}

A. Alteración de la conciencia (p. ej., disminución de la capacidad de atención al
entorno) con disminución de la capacidad para centrar, mantener o dirigir la atención.
B. Cambio en las funciones cognoscitivas (como déficit de memoria, desorientación,
alteración del lenguaje) o presencia de una alteración perceptiva que no se explica por la
existencia de una demencia previa o en desarrollo.
C. La alteración se presenta en un corto período de tiempo (habitualmente en horas o
días) y tiende a fluctuar a lo largo del día.
D. Demostración a través de la historia, de la exploración física y de las pruebas de
laboratorio de que la alteración es un efecto fisiológico directo de una enfermedad
médica, debido a consumo de medicamentos o a intoxicación por alguna sustancia;
síndrome de abstinencia; etiologías múltiples; no especificado

Esta identificación del delirium con el Manual DSM-IV se hace de acuerdo a los criterios anteriormente reflejados en la tabla I, se consideran "delirium" cuando reúnen los dos primeros criterios $(\mathbf{A}+\mathbf{B})$ más alguno de los otros dos, o ambos(C ó $\mathbf{D})$.

La pauta diagnóstica consta de dos elementos: establecer la presencia de delirio (identificar el cuadro) y averiguar la causa enmascarada subyacente para solventarla. 
Algunos autores consideran como características más relevantes del diagnóstico de delirium: la alteración de la conciencia, de la atención, pensamiento desorganizado, con inicio agudo y curso fluctuante (Tabla II) ${ }^{30}$.

Es importante el diagnóstico diferencial con otras patologías que cursan con un cuadro sindrómico semejante al delirum como la demencia, depresión o cualquier otro enfermedad neurológica y/o psiquiátrica frecuente entre los ancianos hospitalizados, para no caer en la confusión clínica y en el error diagnóstico (Tabla II). La Tabla II muestra un cuadro comparativo del Delirum versus Demencia y Depresión por ser ambas patologías bastante usuales entre los ancianos hospitalizados y coexistir con el delirium ${ }^{15}$.

En los pacientes con fractura de cadera se debe indagar sobre el diagnostico diferencial del síndrome de abstinencia y el delirium tremens, en ocasiones muchas de las caídas son secundarias a pacientes bebedores y fumadores habituales.

En la enfermería es primordial que se tenga los conocimientos suficientes para diferenciarlos, identificarlo precozmente, registrarlo y ponerlo de manifiesto al facultativo. La Enfermería son los ojos de la medicina. Hay estudios que destacan la importancia de la valoración de enfermería en la detección precoz del delirium ${ }^{31-35}$.

\section{TABLA II. Delirum Versus Depresión y Demencia ${ }^{36}$}

\begin{tabular}{|l|lll|}
\hline \multicolumn{1}{l}{} & DELIRIUM & DEPRESIÓN & DEMENCIA \\
\hline INICIO & Agudo* & Subagudo & Insidioso \\
CONCIENCIA & Fluctuante* & Estable & Estable \\
ORIENTACIÓN & Alterado* & Vigil & Vigil \\
MEMORIA & Alterada & Intacta & Alterada \\
ATENCIÓN & Deterioro & Conservada & Deterioro \\
DELIRIOS- ALUCINACIONES & Frecuente* & Déficit Parcial & Déficit Parcial \\
\hline
\end{tabular}

* Inouye SK et al. ${ }^{30}$

Existen diferentes instrumentos que ayudan a la detección, diagnostico y cuantificación de la gravedad o el seguimiento de la evolución del delirium ${ }^{37}$. En la tabla III se muestra algunos ejemplos de la gran variabilidad y heterogeneidad de escalas existentes, donde unas son sencillas y otras más complejas (sobrecarga de trabajo). No existe un test específico que mida el riesgo de padecer delirium indistintamente de la causa de hospitalización. El uso de estas herramientas no es necesario para emplear acciones preventivas a grupos de riesgos. 
Delirium. $^{37}$

\begin{tabular}{|c|c|c|}
\hline \multicolumn{2}{|c|}{ DETECCIÓN-DIAGNOSTICO } & CUANTIFICACIÓN \\
\hline $\begin{array}{l}\text {-CAC-A. Clínical Assessment of } \\
\text { Confusión-A.( Vermeersch PE et al. } \\
\text { 1997) } \\
\text {-CRS.Confusión Rating Scale. } \\
\text { (Williams et al. 1988) } \\
\text {-NEECHAM Confusión } \\
\text { Scale.(Champagne y Cols, 1987) } \\
\text {-ICDSC.Intensive Care Delirium } \\
\text { Screening Checklist (Bergeron et al.) }\end{array}$ & $\begin{array}{l}\text { - CAM.Confusional } \\
\text { Assessment Method . } \\
\text { (Inouye et al. 1990) } \\
\text {-DSI.Delirium Symptom } \\
\text { Interview. (Albert et al. } \\
\text { 1992) }\end{array}$ & $\begin{array}{l}\text { - DRS. Delirium Rating Scale.(Trzepacz y } \\
\text { cols,1988) } \\
\text {-CRS.Confusion Rating Scale. (Williams et al. } \\
\text { 1988) } \\
\text {-OBS. Organic Brain Síndrome Scale(Berggren } \\
\text { et al.1987). } \\
\text {-MDAS. Memorial delirium assessment scale. } \\
\text { (Breitbart y Cols. 1997) } \\
\text { - CSE. Confusion State Evaluation (Robertson } \\
\text { y Cols. 1997) }\end{array}$ \\
\hline
\end{tabular}

\section{Etiología. Fisiopatología.}

Su etiología es el resultado multifactorial que surge de la interacción entre la susceptibilidad o vulnerabilidad previa del paciente (Factores Predisponentes internos) y los Factores Precipitantes externos (Desencadenantes) dando lugar a un desorden cerebral ${ }^{4,7,13,38,39}$ (Figura1).

Siendo un síndrome frecuente y común en nuestros hospitales la fisiopatología del delirium no está esclarecida, no se ha profundizado en esta materia, y va a depender en su inmensa mayoría de la etiología que lo genera ${ }^{40}$. Existen muchas conjeturas sobre su fisiopatología por la pluralidad del delirium, en cuanto a sus manifestaciones clínicas como en sus componentes patogénicos, pero según Matías González $\mathrm{T}$ et al. ${ }^{41}$, la hipótesis con mayor peso es la de la "Neurotransmisión", (existe una hipofunción colinérgica con un aumento conjunta de la liberación de la dopamina y serotonina), por lo que la reserva cerebral del paciente determinara la eficacia frente a los agentes agresores del delirium ${ }^{42}$.

\section{Factores de Riesgo: Precipitantes y Predisponentes}

Haciendo un repaso por la bibliografía existente encontramos diferentes factores de riesgos $3,4,10,11,14,26,35,43-67$ predisponentes y precipitantes del delirium (figura 1), sobre cuales basaremos las estrategias de prevención, en los que permitan ser modificados y/o controlados. En ocasiones catalogan a un mismo factor de riesgo como precipitante o predisponente o etiológico, no nos vamos a centrar en esta revisión de la correcta clasificación porque la finalidad de este trabajo es conocer los factores de Riesgo modificables y actuar considerablemente sobre su control.

Aquellos pacientes con una vulnerabilidad previa elevada pueden desarrollar el delirium sin la necesidad de que interaccionen los factores precipitantes ${ }^{35}$, pero generalmente es la combinación de ambos tipos de factores lo que hace aumente la probabilidad de que fluya este mal geriátrico ${ }^{24}$. El anciano hospitalizado con fracturas de cadera por su simple condición posee unos factores de riesgo inherentes a su contexto y situación clínicas, además de aquellos factores individualizados de cada persona, siendo un candidato de alto riesgo: Factores de riesgo propios de pacientes ancianos hospitalizados con fractura de cadera:

- Edad Avanzada.

- Hospitalización (cambio de su hábitat). 
- Escaso personal y constante cambio del personal sanitario (no hay suficiente personal fijo estable) en la unidad.

- Fractura de Cadera.

- Dolor intenso por la patología y movilizaciones por curas, aseos....

- Encamado.

- Intervención quirúrgica ortopédica y estrés.

- Múltiples intervenciones invasivas: canalización de vías, sondajes vesicales...

- Los procedimientos diagnósticos y/o terapéuticos.

- Incomodidad y falta de intimidad para las funciones fisiológicas.

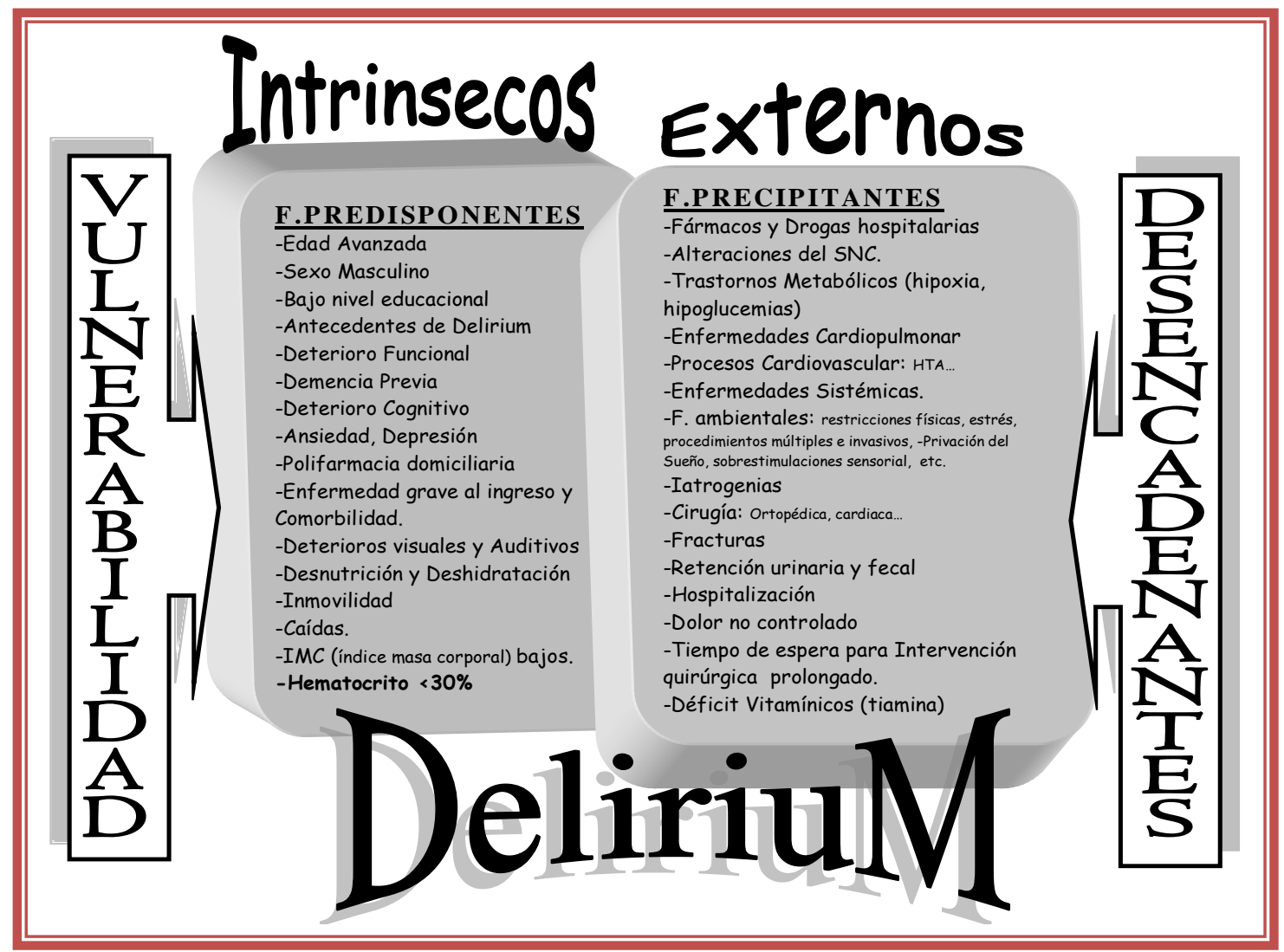

\section{Manifestaciones Clínicas: Signos, síntomas.}

El comienzo agudo y fluctuante en horas y día, acentuándose por la noche, con síntomas variables e intermitentes que se manifiestan por trastorno del ciclo sueño-vigilia, alteración de la memoria a corto plazo, desorientación temporo espacial, distraídos, no prestan atención al medio, ideas paranoides, razonamientos ilógicos, irritabilidad, agitación o letargo, alucinaciones, euforia, ansiedad,... deben de suponer una voz de alarma par la familia, enfermería y el personal sanitario a su cargo ${ }^{4,68}$.

Los síntomas prodrómicos más predictivos en estos pacientes son: "la desorientación" y "Las Ilamadas urgentes para ser atendidos"69.

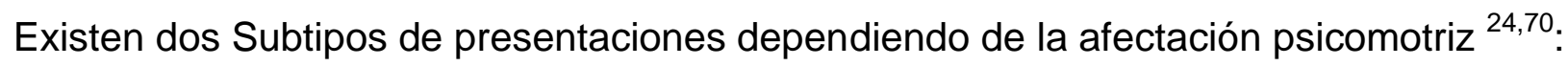


- Hiperactivo: se manifiesta por un estado de agitación psicomotriz, agresividad con el entorno, aumento del estado de alerta e inquietud, Ideas delirantes, alucinaciones, etc. Son más fáciles de diagnosticar por su presentación clínica.

-Hipoactivo: sus manifestaciones son de hipoactividad psicomotora, bajo nivel de conciencia, apatía, letargia, etc. y por su naturaleza son más difíciles de identificar, por lo que no son tratados convenientemente ${ }^{71}$.

-Mixto: Es la mezcla fluctuante entre el hiperactivo e hipoactivo.

Dependiendo del subtipo de delirium, la etiología y la fisiopatología pueden ser diferentes ${ }^{72}$.

\section{La importancia del Delirium. Las consecuencias Negativas. Errores.}

Cuando no se aborda adecuadamente los efectos perniciosos que derivan son de suma importancia $^{73}$ (Tabla IV). El delirium crea un sentimiento degradante en los pacientes consecuencia de la pérdida de razonamiento, facultades y personalidad; la familia sufre la frustración, angustia, ansiedad de la situación aguda a la que se enfrenta.

Se ha demostrado en diferentes estudios que la prevención, el diagnostico precoz y actuación terapéutica en fase inicial minimizan efectos desfavorables y tienden a ostentar mejor evolución ${ }^{25-26,28,53,74-79}$.

A pesar de los recursos existentes no siempre es detectado el delirio por los profesionales sanitarios ${ }^{80-84}$ ni dejamos reflejado en nuestros respectivos registros (graficas de enfermería, historia clínica) la información relevante sobre el delirium ${ }^{85}$.

Existen los errores diagnósticos por confusión con otros trastornos mentales ${ }^{86}$, suelen pasar desapercibidos debido a su presentación clínica ${ }^{87}$ (letargia, somnolencia...) 0 a la complejidad de la misma ${ }^{43}$, no son tratados de forma suficiente ${ }^{47}$. Otro error que perpetramos es no realizar una evaluación cognitiva durante la elaboración de la valoración general del paciente, desconociendo el estado mental previo y sus oscilaciones cognitivas durante su hospitalización ${ }^{35,87}$.

A pesar de que el delirium se considera reversible son pocos los ancianos que al alta hospitalaria salen con el cuadro totalmente resuelto ${ }^{88-90}$; Algunos presentan síntomas residuales, los cuales urgen de un seguimiento continuado y perfeccionado entre la Atención Primaria y Hospitalaria ${ }^{91 .}$

Hay que evitar que se agiten porque son de difícil manejo y están en riesgo de caídas y fracturas. 


\section{TABLA IV Los efectos desfavorables del Delirium ${ }^{3,7,12,23,49,77,86,90,92-100}$.}

1. Elevada morbilidad y mortalidad.

2. Aumento de la rehospitalización.

3. Mayor institucionalización comunitarias o de Larga estancia.

4. Aumenta la estancia hospitalaria con aumento del coste hospitalario y sanitario en general.

5. Mayor empeoramiento Funcional durante la Hospitalización.

6. Peor Evolución y Pronóstico.

7. Mayor riesgo de inseguridad física y psíquica del personal sanitario, familiares y enfermo, compañero de habitación; con repercusiones en la carga de trabajo diaria y en la autoestima profesional.

\section{METODOLOGÍA}

Con el objetivo de investigar sobre la materia a estudio se hizo a priori una búsqueda selectiva de los protocolos, manuales, guías de salud de la práctica clínica, libros en formato electrónico y papel, utilizando bibliotecas virtuales o físicas y sus consiguientes páginas de internet donde se encuentran ubicadas esta información (FISTERRA, MEDLINE, GUIASALUD, GOOGLE LIBROS, BV-SSPA, LIBRO DSM-IV-TR, etc.).

En un segundo lugar se realizado una búsqueda bibliográfica con diferentes descriptores en la base de datos $\mathrm{MeSH}$, hasta optar por las que definen la materia a estudio. Fruto de los descriptores se investiga de forma metódica en las variadas bases de datos biomédicas con la combinación de los operadores booleanos (AND, NOT, OR): PubMed, IBECS, LILACS, TESIDE, MEDLINE, Cuiden, SciElo España y Brasil, La biblioteca Cochrane pus, Google Académico; Revistas de psicología, geriatría y gerontología, sociedades científicas.

En "Single Citation Matcher" se ha hecho búsqueda selectiva de los autores más destacados y de forma manual en las diferentes referencias de los estudios seleccionados.

Los criterios de inclusión son artículos originales de pacientes ancianos hospitalizados en diferentes unidades clínicas (preferentemente con fracturas de cadera) donde incluyen datos sobre la prevención no farmacológica del delirium y la implicación de la enfermería, en idioma español e inglés, artículos completos y su resumen. Sin límite en la búsqueda: en el año de publicación y distribución geográfica.

\section{RESULTADOS Y DISCUSIÓN}

\section{Estrategias de Actuaciones Preventivas: Recomendaciones de Enfermería.}

La estrategia en el tratamiento reúne tres vías de acción: prevención, tratamiento de la enfermedad de base, y manejo sintomático del delirium (Medidas generales y farmacológicas). Nos centramos mayoritariamente en la prevención primaria como medida eficaz y económica en el manejo del delirium.

Diferentes estudios avalan la eficacia de las intervenciones preventivas por parte de enfermería en el manejo del delirum, entre los que se encuentran: 


\section{Pacientes de Cirugía Cardiaca y Medicina General:}

-Lazarus HR et al ${ }^{101}$ en 1968 con 54 pacientes de cirugía cardiaca consiguió reducir la frecuencia total del delirium postoperatorio en un $19 \%$, con recomendaciones individuales en el cuidado postoperatorio, y disminuyendo los dificultades ambientales de la sala de recuperación.

-Budd S et al ${ }^{102}$ analizó en 1974 a 31 pacientes programados para cirugía cardiaca entre 23-63 años, alcanzado una significativa reducción del $81 \%$ del delirium postoperatorio con tácticas para la reorientación del paciente en tiempo, lugar, persona, y mejoras del estado físico.

-Chatham MA ${ }^{103}$ en 1978 estudió a un reducido grupo de 20 pacientes quirúrgicos, donde las intervenciones iban orientadas a la educación al paciente y cuidador principal, obtuvo resultados ligeramente favorables en cinco de las once áreas de intervenciones llevadas a cabo.

-Owens JF y Hutelmyer $\mathbf{C M}^{104}$ en 1982 investigó a 64 pacientes cirugía cardiaca, consiguiendo con intervenciones de educación hacia el pacientes reducir la frecuencia del delirium en el preoperatorio con un total del $19 \%$.

-Wanich CK et al ${ }^{105}$ con un grupo más amplio de 235 pacientes de medicina genera, en 1992 y con intervenciones dirigidas a la educación del personal sanitario, orientación, movilización, modificaciones ambientales y evaluación de los tratamientos consiguió minimizar el delirium total en un $3 \%$.

-Inouye SK et al ${ }^{56}$ en 1999 estudio a 852 pacientes, mayores de 70 años admitidos en medicina general y demostró que las estrategias de prevención primaria en el control de seis factores de riesgo específico (deterioro cognitivo, inmovilidad, discapacidad visual y auditiva, deshidratación y privación del sueño) originaron una reducción del $40 \%$ el riesgo de padecer el delirium, concluye e su estudio que la educación primaria y la prevención es factiblemente la estrategia más efectiva para afrontar el delirium.

-Tabet $\mathbf{N}$ et al ${ }^{106}$ en el 2005 expuso con un programa educacional económico dirigido al personal sanitario una reducción de la prevalencia del delirium de un 19,5\% a 9,8\% en ancianos mayores de 70 años (población a estudio de 250 pacientes de medicina interna).

-Lundström $\mathbf{M}$ et $\mathbf{a l}^{107}$ en abril del 2005 originó un descenso en la estancia hospitalaria, en la duración del delirium y en la mortalidad en 400 pacientes mayores de 70 años a partir de intervenciones de educación hacia el personal sanitario y reorganización de la atención hacia el paciente (enfermería y medicina).

-En Francia Gentric A et al ${ }^{108}$ en 2007, con una población de pacientes mayores de 75 años llegó a reducir la frecuencia del delirium en una unidad de medicina interna tras aplicar medidas preventivas.

-Vidan MT et al ${ }^{109}$ recientemente (2009), realizo en un Hospital de Madrid con 542 pacientes de Medicina interna, mayores de 70 años, un programa preventivo no farmacológico con intervenciones educativas y acciones específicas sobre las áreas de: orientación, sensorial, sueño, movilización, hidratación, nutrición, consumo de drogas. Reduce el delirio sin costes adicionales sanitarios y mejora la calidad asistencial. 


\section{Pacientes de cirugía ortopédica:}

-Williams MA et al ${ }^{110}$ en 1985 trabajó con 227 pacientes con fractura de cadera mayores de 60 años, proporcionó como resultado de las intervenciones preventivas (educación al paciente, medidas de orientación y apoyos sensoriales) en el pre y postoperatorio una caída del total de la frecuencia del delirium del $8 \%$.

-Strömberg L et al ${ }^{111}$ en 1999, en Suecia, valoró medidas de reorientación en el perioperatorio a 223 pacientes con fractura de cadera (81 años de media), la conclusión declinaba la importancia del entorno psicológico del paciente.

-Lundström $\mathbf{M}$ et al $^{112}$ en 1999 trabajo con un grupo de pacientes con fractura de cuello femoral, con un programa de intervenciones en el postoperatorio, abarcando las áreas de educación al personal sanitario, cooperación entre cirujanos ortopédicos y Geriatras, atención individual y planificación de rehabilitación, mejora del entorno ambiental, nutrición adecuada, mayor continuidad de la atención, prevención y tratamiento de complicaciones asociadas con delirio, alcanzó una reducción reveladora de la incidencia del delirium en comparación con otros estudios previos, además se acortó la duración de delirio y se mejoró la funcionalidad de los anciano tratados por fracturas de cuello femoral.

-Milisen $\mathbf{K}$ et $\mathbf{a l}^{53}$ en 2001 presento un estudio de 120 pacientes, en Suecia, con intervenciones en educación hacia enfermería, evaluación cognitiva metódica, protocolos de control del dolor, disponibilidad de médico o enfermera especializado en geriatría, consiguió reducir la duración y gravedad del delirium, sin hallar una disminución relevante en la incidencia.

-Björkelund KB et $\mathbf{a l}^{113}$ en un artículo publicado en 2010 reduce la incidencia de delirium en un $35 \%$ en pacientes ancianos lúcidos con fractura de cadera con ocho intervenciones aplicadas en el preoperatorio (Oxigenación y fluidos intravenosos suplementarios, control de contantes y dolor, evitar demora logística de transferencia, escala diaria OBS (escala de síndrome cerebral orgánico), evitar la polifarmacia, adecuado periodo perioperatorio y anestésico).

También existen publicaciones donde los resultados de las intervenciones preventivas no demuestran generar efectividad significativa, como sucede en el estudio de 60 pacientes realizado por Nagley S $\mathbf{J}^{114}$ en 1986; Siddiqi $\mathbf{N}$ et al ${ }^{115}$ en el 2008 realiza un revisión con el objetivo de conocer la efectividad de las intervenciones diseñadas para prevenir el delirio en los pacientes hospitalizados dando escasos resultados y la necesidad de realizar mayores estudios en este ámbito.

Otra línea de trabajos son aquellos artículos que aunque no basan sus estudios en expresar la eficacia de adoptar medidas preventivas apuntan intervenciones apoyándose en el control de los factores de riesgo modificables ${ }^{4,25-27,32,78,116-121}$

Existen protocolos estandarizados en diferentes hospitales que mejoran la calidad de los cuidados en pacientes hospitalizados (previene el deterioro funcional y cognitivo), un precedente de ellos es el "Hospitla Elder Life Program ${ }^{122}$ (HELP)", que abarca un Programas de intervenciones en las aéreas de movilidad, alteraciones sensoriales y auditivas, orientación, nutrición, hidratación y ritmo sueño-vigilia para aplicar medidas 
preventivas; Este programa es único en su enfoque y se ha implantado en más de 30 hospitales en todo el mundo.

No existe actualmente una intervención aislada que nos exima del delirium ${ }^{49}$, ante una misma exposición a factores de riesgos la respuesta puede ser diferente ante distintos pacientes.

Los factores de riesgos en pacientes ancianos con fractura de cadera van a variar dependiendo si se hallan en el preoperatorio o postoperatorio, por lo que habría que definir estrategias diferentes para su prevención ${ }^{10}$.

Se da una discrepancia en los resultados obtenidos por las diversas publicaciones, donde los rangos de porcentaje son dispares, esto puede deberse a diferentes: método de estudio, población (tipo y número de pacientes), momentos clínicos (preoperatorio, postoperatorio), variables (Factores de riesgo versus Medidas preventivas), método diagnóstico de evaluación, adhesión al protocolo de intervenciones, medidas de frecuencia (prevalencia, incidencia) y el infradiagnóstico del delirium, sobre todo el hipoactivo.

Las siguientes recomendaciones se asientan en base a las revisiones literarias existentes sobre los factores de riesgos asociados al delirium, indicaciones de intervenciones preventivas ambientales y no farmacológicas, los artículos que manifiestan la eficacia de medidas preventivas sobre ciertos factores relacionados con mayor riesgo de esta patología, además de aportar la modesta experiencia personal como enfermera en unidad de traumatología ortopédica.

Tabla V. Estrategias Preventivas de Enfermería en Traumatología: Intervenciones Ambientales y terapéuticas ante Factores de Riesgo potencialmente modificables en el delirum. Fractura de Cadera. Anciano.

\section{RECOMENDACIONES GENERALES PREVENTIVAS DE ENFERMERÍA PARA} ANCIANOS HOSPITALIZADOS CON FRACTURA DE CADERA

\section{Intervenciones Ambientales-entorno/estimulación-Hospitalización}

Adecuar un entorno seguro. Aplicar Protocolo de prevención de Caídas. Barandillas. Bastones, andadores, zapatos cerrados en la deambulación, etc.).Retirar objetos potencialmente lesivos e innecesarios. Cama adaptada a una altura adecuada. Valorar riesgo caídas con test.

$\checkmark$ Colocar Colchón antiescaras a todo anciano con Fx de Cadera.

$\checkmark$ Disponerle un adecuado Confort en la Cama. Sabanas sin pliegues, los sistemas de soporte que no interfieran en la comodidad, etc.

$\checkmark$ Mantener una temperatura ambiental confortable. Habitación con ventanas.

$\checkmark$ Designarle una habitación con otro enfermo en caso de no tener cuidador principal y cerca del control de enfermería para mayor vigilancia. (evitar aislamiento social). Evitar colocar a dos pacientes con alto riesgo o con delirium establecido en la misma habitación. 
$\checkmark$ Disponer de habitación individual, en función de los recursos disponibles, para fomentar el descanso y la tranquilidad si tiene el enfermo acompañante (familiar).

$\checkmark$ Adecuada iluminación en la habitación y tranquila. Iluminación indirecta durante la noche. Evitar la sobre estimulación sensorial

$\checkmark$ Evitar ruidos externos e internos. (No: Televisión con alto volumen, hablar fuerte...). Colocar carteles de "silencio" en cada habitación.

$\checkmark$ Evitar trasiego constante del personal sanitario en la habitación.(agrupar intervenciones)

$\checkmark$ Evitar estar muchos familiares en la habitación y personas ajenas a la misma. Establecer horarios de visitas y máximo de dos personas por paciente-habitación. Colocar carteles informativos de las normativas de la planta en la habitación.

$\checkmark$ Permitir el uso utensilios propios no peligrosos.(almohadas, sabanas, camisones...)

$\checkmark$ Respetar y Valorar un apropiado Ciclo de sueño-vigilia. Utilizar Protocolo de medidas no farmacológicas a priori para favorecer el sueño nocturno (bebidas templadas, infusiones relajante....) e instruir al familiar en ellas. Las medicaciones e intervenciones terapéuticas no deben interrumpir el sueño. Planificar actuaciones preservando los periodos de descanso del paciente. Preguntar al paciente o familiar cómo ha descansado diariamente.

$\checkmark$ Aplicar protocolo para el manejo no farmacológico de la Ansiedad. Técnicas de relajación, etc.

\section{Orientación}

Informarle al ingreso de la distribución y recursos de lo que se dispone en la habitación y la unidad de traumatología. (luces, baños-duchas, bateas, toallas, dispositivos de llamadas al personal (timbres), enchufes, teléfonos, ...)

$\checkmark$ Mantener un personal sanitario estable y habitual en la unidad de traumatología para una mejora en la atención individualizada y mayor familiaridad con el paciente.

$\checkmark$ Permitir y Fomentar el uso de relojes, calendarios, periódicos, revistas, televisores, pasatiempos, fotos, gafas, audífonos, prótesis o cualquier objeto que promueva su orientación.

$\checkmark$ Colocar un reloj de pared por habitación con numeración visible y clara.

$\checkmark$ Colocar objetos familiares al alcance visual del enfermo.

$\checkmark$ Evitar cambiarlo de habitación y de reubicación en otras unidades hospitalaria durante el ingreso por necesidades de disponibilidad de camas ante urgencias hospitalarias o por precisar estar algún día en UCI (reservarle la misma habitación) 
$\checkmark$ Durante su traslado para la realización de pruebas diagnósticas o terapéuticas que sea acompañado el cuidador principal.

$\checkmark$ Distribuir la habitación, adaptadar a sus preferencias en lo que sea viable. Respetar un espacio considerable entre los pacientes cuando se comparte habitación.

$\checkmark$ Fomentar e instruir al cuidador principal en la orientación: Crear un programa de actividades orientativas (tablas de orientación, horarios, agendas, etc.). Tres veces al día.

\section{Familia-Cuidador principal}

$\checkmark$ Instruir y Educar al cuidador principal en el manejo de la seguridad, movilizaciónRehabilitación, orientación (tabla de orientación, horarios, etc.), distracción, estimulación cognitiva (reminiscencia, juego de palabras, hechos presentes, etc.), manejo no farmacológico del sueño y estrés, importancia de limitaciones de visitas al paciente...

$\checkmark$ Contactar con la Asistente Social cuando se precise, para proporcionarles recursos sociales convenientes. Ej: Buscarle un cuidador durante la estancia hospitalaria.

$\checkmark$ Contactar con la Enfermera de Enlace para su seguimiento y posterior conexión con Atención Primaria(optimización de recursos)

$\checkmark$ Implicar a la familia en el abordaje del delirium y cuidados del paciente. Educación y asesoramiento familiar sobre él y las técnicas necesarias para su prevención (Ej: Técnicas de relajación y autocontrol para reducir estrés y/o ansiedad del paciente, formar en la estimulación cognitiva durante el día)

$\checkmark$ Aconsejar pautas de actuación ante la aparición del delirium e instruir sobre la identificación de signos prodrómicos de alerta: Gritos, Inquietud (tocarse constantemente sondas, vías....), Hipoactividad, Dificultad para dormir o alteración vigilia-sueño, agresividad verbal y/o física, Irritabilidad, Labilidad emocional, Alucinaciones y no reconocimiento del lugar (donde se encuentra) ni del familiar, etc.

$\checkmark$ Informar a la familia de forma clara sobre todas aquellas inquietudes que pueda tener. Apoyo emocional. Escucha Activa. Estimular a la familia y al cuidador a expresar sus sentimientos, frustraciones y problemas.

\section{Estado Cognoscitivo}

$\checkmark$ Valorar estado Mental al ingreso, durante la hospitalización si se produce oscilaciones en su estado mental y al alta. ESCALAS (Peeiffer, Mini-Mental State Examination...).

$\checkmark$ Fomentar e instruir al cuidador principal en la estimulación cognitiva: Crear un programa de actividades terapéuticas Cognoscitivas (pasatiempos, juegos de adivinanza, palabras, etc.). Tres veces al día. 
Valoración de la Capacidad funcional al ingreso, durante la hospitalización y al alta. ESCALAS (katz, Barther, Escala Cruz Roja...)

$\checkmark$ Fomentar y favorecer que el paciente participe de sus cuidados. Mantener en lo posible su autonomía e independencia.

\section{Comunicación verbal-no verbal}

$\checkmark$ La información facilitada debe ser objetiva, adaptada a un lenguaje claro y comprensible, teniendo en cuenta el nivel cultural, sobre su diagnostico, tratamiento y pronóstico. Asegurarnos de que ha comprendido y escuchado la información. Repetir cuantas veces sea necesaria en un tono suave. Evitar "jergas médicas".

$\checkmark$ Utilizar adecuada comunicación verbal y no verbal. Contacto visual y físico. Escucha activa. Trato amable, paciente.

$\checkmark$ Escuchar al paciente y familiares e incluirlos en la conversación. Resolverles dudas e inquietudes en todo momento.

$\checkmark \quad$ Evitar actitudes hostiles.

$\checkmark$ Presentarse al paciente y dirigirse a él como una persona adulta y por su nombre. Tarjetas identificativas en todo el personal de la unidad.

$\checkmark$ Proporcionar un traductor en pacientes extranjeros que no manejen el idioma o un intérprete si fuese necesario para su comunicación.

$\checkmark$ Adaptar el medio ambiente para pacientes con discapacidad visual y/o auditiva

\section{Actuaciones Terapéuticas de Enfermería y de Colaboración con el Equipo Multidisciplinario}

Ingresar al paciente con la mayor brevedad a la unidad de traumatología cuando su ingreso procede de las urgencias hospitalarias (evita estrés, aglomeraciones, sobre estimulaciones sensoriales derivadas de la unidad de urgencia, etc.).

$\checkmark$ Realizar entrevista al paciente y/o cuidador principal, Valoración de enfermería, registro de todos los datos bio-psico-social en la grafica de enfermería (Escala de cribaje: CAM..). Generar un Plan de Actuaciones integral de enfermería individualizado y específico. Indagar sobre el síndrome de abstinencia o delirium tremens.

$\checkmark$ Aplicar protocolo de Seguimiento, prevención y/o tratamiento de UPP.

$\checkmark$ Aplicar protocolo de Prevención de infecciones nosocomiales: neumonías, infecciones urinarias, etc.

$\checkmark$ Manejo Eficaz del Dolor. Valorar control adecuado del dolor (Ej: EVA (Escala 
valoración Analógica)...).Identificar la etiología del dolor y subsanarla con la mayor brevedad (revisar tracción cutánea, férula de Braun, protección de talones, etc.). Protocolo del Manejo del Dolor.

$\checkmark$ Evitar en todo lo posible la restricción física, y si se aplica, de forma segura y con vigilancia constante del paciente para prevenir complicaciones (ulceras, asfixia, éstasis venos, etc.)

$\checkmark$ Movilización temprana. Ejercicios de movilización pasivos en cama (preoperatorio). Sedestación y deambulación temprana tras postoperatorio.

$\checkmark$ Rehabilitación inmediata. Iniciación inmediata desde la unidad de traumatología (enseñar ejercicios de rehabilitación) y posteriormente enlazar en colaboración con la unidad de Rehabilitación hospitalaria.

$\checkmark$ Identificar y Manejar posible aparición del Sundowning (Síndrome del crepúsculo): tirar objeto, coger y hurgar con ropa, vociferaciones y musitaciones, constante demanda del personal sanitario, etc.

$\checkmark$ Agrupar acciones terapéuticas (Ejemplo: canalizar vía y extraer analíticas a la vez, Realizar curas de lesiones cutáneas al movilizar al paciente durante la higiene diaria, etc.) minimizando el número de intervenciones terapéuticas y trasiego en la habitación.

$\checkmark$ Asegurar el correcto funcionamiento de los soportes para evitar reintervenciones al paciente por Iatrogenias o accidentes (Vigilar, asegurar y fijar sondajes, drenajes, vías, tracciones...etc.)

$\checkmark$ Mantener o Restablecer buen estado nutricional. Incentivar la ingesta alimenticia y adaptarla individualmente al paciente (dietas blandas hiperproteica, suplementos dietéticos, aportes vitamínicos (Grupo B) y ricas en fibra para prevenir estreñimiento, trituradas etc.). Colocar dentaduras si no está contraindicado. Identificar signos de desnutrición (IMC...). Pesar, tallar, circunferencia del brazo, pliegues cutáneos...

$\checkmark$ Mantener o Recuperar un buen estado de hidratación (fomento y control de la ingesta hídrica, aplicar cremas hidratantes), Detección precoz de signos de deshidratación (cefaleas, poca diuresis y concentrada, piel desestructurada, etc.).

$\checkmark$ Fomentar, Manejar y Vigilar un adecuado funcionamiento vesical e intestinal. Medidas protectoras ante el estreñimiento e incontinencia vesical. Dar intimidad para defecar y miccionar. Reeducación vesical tras retirada de sondaje vesical.

$\checkmark$ Mantenimiento y Vigilancia de buen Control hidroeléctrolítico. Realizar balance hídrico diario. Identificación precoz de signos y síntomas de alteraciones Hidroelectrolíticas. 
Identificar y Manejo precoz de síntomas prodrómicos (irritabilidad, gritos, agresión verbal...)

$\checkmark$ Vigilancia, identificación temprana y control del Factores de riesgo del delirium. Realizar la escala CAM para el diagnostico precoz del delirium.

$\checkmark$ Administrar mantas tras postquirofano, evitando la hipotermia.

$\checkmark$ Control y Manejo de Constantes Vitales-Hemodinámica preoperatorias y postoperatoria por turno de trabajo y cuando se precise:

a. Medir la Saturación (pulsioximetría) según prescripción médica y cuando se sospeche hipoxia. Colocar oxigenoterapia según prescripción facultativa en caso de trastornos respiratorios para mantener una Saturación $>90 \%-95 \%$.En el postoperatorio oxigenoterapia durante $48 \mathrm{~h}$ tras intervención quirúrgica.

b. Medir la Tensión Arterial según prescripción médica y cuando se sospeche signos de alteraciones en la tensión arterial, FC o temperatura corporal. (Se aconseja mantener las TAS $>90 \mathrm{mmHg}$.

Control de Glicemias en pacientes diabéticos y con periodos largos de ayunos diagnósticos y/o terapéuticos. Proceder con protocolo de diabéticos, evitar hipo e hiperglucemias.

$\checkmark$ Realización de analíticas bajo prescripción médica ante sospechas de alteraciones.

$\checkmark$ Optimizar la pronta realización de cualquier procedimiento diagnóstico y terapéutico.

$\checkmark$ Fisioterapia Respiratoria durante el tiempo de inmovilización en la cama.

$\checkmark$ Vigilancia e Identificar signos de hipovolemia (Aconsejan mantener hematocrito >30\%)

$\checkmark$ Identificación precoz de signos y síntomas de complicaciones pre y postoperatorias potenciales precipitantes del delirium (alteraciones metabólicas, trastornos vasculares, hipoperfusión, infecciones, embolia grasa, etc.).

$\checkmark$ Revisión diaria y meticulosa de la Medicación tanto hospitalaria como domiciliaria conjuntamente con el Cirujano Ortopédico, eliminando y/o reduciendo dosis farmacológicas innecesarias, así como la revalorización de aquellas intervenciones terapéuticas de soportes sobrantes (retirada drenajes, sondajes, vías venosas, pañales, etc.). No olvidar valorar la posibles ingesta de hierbas medicinales domiciliarias.

Estas recomendaciones deben ir acompañada de un seguimiento continuado y vigilancia constante del paciente por todo el equipo multidisciplinario implicado en su proceso y la familia. Se debe comprobar diariamente la adherencia de aquellas actividades programadas hacia la prevención del delirium. Sería interesante crear folletos informativos donde se bride unos consejos a los familiares para el manejo de los pacientes de riesgo de delirium. 


\section{CONCLUSIONES}

El delirum es un patología neuropsiquiatría aguda, severa, en ocasiones infradiagnosticada, de etiología multifactorial con base orgánica (enfermedad subyacente), prevenible, reversible, aunque suele dejar secuelas cognitivas, y con una alta frecuencia sobre todo a nivel hospitalario y en ancianos con fractura de cadera. Con relevante impacto negativo a nivel bio-psico-social en la integridad del paciente y su entorno unido a las repercusiones económicas socio-sanitarias que genera. Cabe destacar los efectos potenciales en la inseguridad física y psíquica del personal sanitario y la sobrecarga de trabajo diaria que genera.

La gran variabilidad de este síndrome hace que enfermos con los mismos factores predisponentes presenten cuadros clínicos desiguales, danto complejidad a las estrategias de actuación.

La Enfermería juega un papel fundamental en la detección, prevención, diagnostico y tratamiento precoz por la estrecha relación continuada e integral con el paciente y la familia. Es importante el tener unos conocimientos y habilidades esenciales para llevar a cabo esta labor multidisciplinaria creando programas de educación, estableciendo Protocolos y Guías de la Práctica Clínica acorde a la realidad y medios económicos disponibles, unificando criterios de intervención sanitaria para reducir la variabilidad y asegurar una correcta actuación terapéutica por todo el equipo multidisciplinario en la práctica clínica.

La falta de apreciación del médico como una emergencia médica y de no dársele la importancia adecuada por el personal sanitario y los organismos responsables hacen que no se ponga en marcha los dispositivos adecuados para erradicar o disminuir su elevada magnitud.

Las intervenciones preventivas es la estrategia eficaz, eficiente y económica para el control del delirium, no existe una intervención aislada sino un conjunto de medidas preventivas para combatirlo dependiendo del momento clínico (preoperatorio-postoperatorio) y de los factores inherentes del paciente y al contexto.

El Rol de la enfermería dentro del equipo multidisciplinario reside en la valoración integral del enfermo, la entrevista con los familiares y paciente, la valoración del riesgo y repercusiones del delirium en el entorno del paciente, la detección, el cumplimiento y seguimiento de los diagnósticos de enfermería, la planificación de las intervenciones y evaluación de los resultados obtenidos en los cuidados individualizados, la colaboración terapéutica interdisciplinaria, la preparación del alta con un informe de continuidad de cuidados y recomendaciones, además de la coordinación con los recursos intrahospitalarios (unidades de media y de larga estancia) y extrahospitalarios (residencias y atención primaria).

No siempre podemos hacer lo que es mejor para el paciente por las limitaciones físicas, humanas y económica a la estamos sometidos, sería necesario un llamamiento a las instituciones correspondiente para que tomen medidas encaminadas a la prevención de este gran síndrome, ya que en sí, constituye un marcador de la calidad de los servicios sanitarios. Sería interesante en otros trabajos futuros valorar la eficacia de las recomendaciones ofrecidas en esta revisión. 


\section{BIBLIOGRAFÍA}

1. Morandi A, Pandharipande P, Trabucchi M, Rozzini R, Mistraletti G, Trompeo AC, et al. Understanding international differences in terminology for delirium and other types of acute brain dysfunction in critically ill patients. Intensive Care Med. 2008;4:1907-15.

2. Johnson MH. Assessing confused patients. J Neurol Neurosurg Psychiatry. 2001;71:7-12.

3. Formiga F, Marcos E, Solé A, Valencia E, Lora-Tamayo J, Pujo IR. Síndrome confusional agudo en pacientes ancianos ingresados por patología médica. Rev Clin Esp. 2005;205:484-8.

4. Inouye SK. Delirium in older persons. N Engl J Med. 2006;354:1157-65

5. Leslie DL, Marcantonio ER, Zhang Y, Leo-Summers L, Inouye SK. One-year health care costs associated with delirium in the elderly population. Arch Intern Med. 2008;168:27-32.

6. Ribera Casado J. El síndrome confusional agudo: un Síndrome Geriátrico en alza. Revista Clínica Española. 2005;205:469-71.

7. Guillén Llera F. Delirium en pacientes ancianos hospitalizados. Med Clin (Barc). 2005;124:538-40.

8. Laurila JV, Pitkala KH, Strandberg TE, Tilvis RS. The impact of different diagnostic criteria on prevalence rates for delirium. Dement Geriatr Cognit Disord. 2003;16(3):156-61

9. Norberg Y Gustafson, Berggren D, Brannström B, Bucht G, A, Hansson me, et al. Acute confusional states in elderly patients treated for femoral neck fracture. J Am Geriatr Soc. 1988; 36(6):525-30.

10. Edlund A, Lundström M, Brannström B, Bucht G, Gustafson y. Delirium before and after operation for femoral neck fracture. J Am Geriat Soc. 2001:49:1335-40.

11. Formiga F, Rier-Mestre A, Chivite D, Pujol R. Delirium en pacientes mayores de 94 años con fractura de fémur. Rev Esp Geriatr Gerontol. 2008;43(3):154-6

12. Brauer C, Morrison RD, Silberzweig SB y AL Siu. The cause of delirium in patients with hip fracture. Arco Intern med. 2000;160(12):1856-60

13. Altimir S, Prats M. Síndrome confusional en el anciano. Med Clin (Barc). 2002;119(10):386-9.

14. Ribera Casado JM, Cruz Jentoft AJ (Ed). Geriatría en Atención Primaria, 3ª edición. Barcelona: Aula Médica, 2002.

15. Lipowski ZJ. Delirium in the elderly patient. N Engl J Med 1989; 320: 578-82.

16. Sáez López P, Madruga Galán F, Rubio Caballero JA. Detección de problemas en pacientes geriátricos con fractura de cadera. Importancia de la colaboración entre traumatólogo y geriatra. Rev Ortop Traumatol.2007;51:144-51

17. O'keeffe st, NI Chonchubhair A. Postoperative delirium in the elderly. British Journal of Anaesthesia. 1994; 73: 673-87.

18. Cofer M.J. Un compañero inoportuno del anciano: el delirio postoperatorio. Nursing. 2006; 24(1):21-23.

19. Benjamin D. Robertson and Timothy J. Robertson. Postoperative Delirium After Hip Fracture .Bone Joint Surg Am. 2006;88:2060-2068.

20. Rothschild JM, Bates DW, Leape LL. Preventable medical injuries in older patients. Arch Intern Med. 2000;160:2717-28.

21. Inouye SK. Delirio: Un barómetro para la calidad de la asistencia hospitalaria. Hospital Practice. 2002;1(6): 52-55.

22. Carretero Faner, M. Plan de cuidados: síndrome confusional agudo. Gerokomos. 2002 dic. 13(4): 178-184.

23. Delirium, demencia, trastornos amnésicos y otros trastornos cognoscitivos. In: LópezIbor Aliño JJ, Valdés Miyar M, editors. DSM-IV-TR. Manual diagnóstico y estadístico de Ios trastornos mentales: texto revisado. Barcelona: Masson; 2005.

24. Textbook of Consultation-Liaison Psychiatry, $2^{\underline{a}}$ ed. Washington DC. The American Psychiatric Publishing; 2002. 
25. Sánchez I. Tratamiento del delirium. Rev Psiquiatría Fac Med Barna 2000; 27(5): 279284

26. Bekker AY, Weeks EJ - Cognitive function after anaesthesia in the elderly. Best Pract Res Clin Anaesthesiol. 2003;17:259-272.

27. Meagher DJ. Delirio: optimización de la gestión.BMJ.2001;322:144-49.

28. Moreno Rodríguez Ana. El delirio en enfermos con fractura de cadera, su incidencia. Enferm. glob. [revista en la Internet]. 2010 Oct [citado 2011 Jun 08] ; (20): Disponible en: $\quad$ http://scielo.isciii.es/scielo.php?script=sci_arttext\&pid=S169561412010000300005\&lng=es. doi:10.4321/S1695-61412010000300005

29. Blanco López B, Cruz Niesvaara D, Ibero Villa JL, Sardó Pascual E. Delirium:diagnóstico y tratamiento. Medicina General. 2004;67:491-496.

30. Inouye SK, Van Dyck $\mathrm{CH}$, Alessi CA, et al. Clarifying confusion: the confusion assessment method. A new method for detection of delirium. Ann Intern Med.1990;113:941-8.

31. Claver Martín MD. Actualización en la evaluación y tratamiento del delirium. Rev Esp Geriatr Gerontol. 1998;33:227-35.

32. Cid M. Cuadros confusionales agudos. En: Guillen F, Pérez J, editores. Síndromes y cuidados en el paciente geriátrico. Barcelona: Masson; 1994. P. 211-6.

33. Beers MH, Berkow R. Manual Merck de geriatría. 2.a ed. Madrid: Harcout; 2001.

34. Vermeersch PEH. The clinical assessment of confusion. Appl Nurs Res. 1990;113:12833.

35. Inouye SK, Charpentier PA. Precipitating factors for delirium in hospitalized elderly persons: predictive model and interrelationships with baseline vulnerability. JAMA. 1996;275:852-7.

36. Pérez Martínez Víctor T.. Demencias: su enfoque diagnóstico. Rev Cubana Med Gen Integr [revista en la Internet]. 2006 Dic [citado 2011 Oct 16] ; 22(4): . Disponible en: http://scielo.sld.cu/scielo.php?script=sci arttext\&pid=S086421252006000400010\&lng=es.

37. Muñoz Rodríguez PE. Test de Cribado en la práctica clínica. En: Bulbena Vilarrasa A, Berrios E G, Fernández de Larrinoa Palacios P, editores. Medición clínica en psiquiatría y psicología. Barcelona: Masson; 2000. p. 35-51.

38. Barrero Raya MC, Parras García de León N. Delirium en el anciano. Salud Rural. 2001; XVIII (12): 11-49.

39. Roche V. Southwestern Internal Medicine Conference. Etiology and management of delirium. Am J Med Sci.2003; 325 (1): 20-30.

40. Meagher JM. Delirium: optimizing management. BMJ .2001; 322: 144-50.

41. Tejeiro Martínez J, Gómez Sereno B. Guía diagnóstica y terapéutica del síndrome confusional agudo. Revista Clínica Española. 2002; 202 (05):280-288.

42. Jones RN, Fong TG, Metzger E, Tulebaev S, Yang FM, Alsop DC, et al. Aging, brain disease, and reserve: implications for delirium. Am J Geriatr Psychiatry. 2010;18:117-27.

43. González M, De Pablo J, Valdés M. Delirium: la confusión de los clínicos. Rev Méd Chile. 2003; 131:1051-60

44. Galanakis $P$, Bickel H, Gradinger R, Von Gumppenberg S, Forstl H: Acute confusional state in the elderly following hip surgery: incidence, risk factors and complications. Int $J$ Geriatr Psychiatry. 2001;16:349-355.

45. Gustafson Y, Berggren D, Brannstron B, et al. Acute confusional states in elderly patients treated for femoral neck fracture. J Am Geriatr Soc. 1988;36:525-30.

46. Edlund A, Lundstrom M, Lundstrom G, et al: Clinical profile of delirium in patients treated for femoral neck fractures. Dement Geriatr Cogn Disord.1999;10:325-29.

47. Cole MG. Delirium in Elderly Patients. Am J Geriatr Psychiatry. 2004;12:7-21. 
48. Marcantonio ER, Goldman L, Orav EJ, Cook EF, Lee TH. The association of intraoperative factors with the development of postoperative delirium. Am $\mathrm{J}$ Med. 1998;105:380-4.

49. Marcantonio ER, Flacker JM, Michaels M, Resnick NM. Delirium is independently associated with poor functional recovery after hip fracture. J Am Geriatr Soc.2000;48:618-24.

50. Chrispal A, Mathews KP, Surekha V. The clinical profile and association of delirium in geriatric patients with hip fractures in a tertiary care hospital in India. J Assoc Physicians India. 2010;58:15-9.

51. Morrison RS, Magaziner J, Gilbert M, Koval KJ, McLaughlin MA, Orosz G, Strauss E, Siu AL. Relationship between pain and opioid analgesics on the development of delirium following hip fracture. J Gerontol A Biol Sci Med Sci. 2003;58:76-81.

52. Adunsky A, Levy R, Heim M, Mizrahi E, Arad M. The unfavorable nature of preoperative delirium in elderly hip fractured patients. Arch Gerontol Geriatr. 2003;36:67-74.

53. Milisen K, Foreman MD, Abraham IL, De Geest S, Godderis J, Vandermeulen E, et al. A nurse-led interdisciplinary intervention program for delirium in elderly hip-fracture patients. J Am Geriatr Soc. 2001;49:523-32.

54. Gotor P, González JI, Alarcón MT. Factores asociados a la aparición del delirium en pacientes geriátricos con fractura de cadera. Rev Mult Gerontol. 2004;14(3):138-48.

55. Elie M, Cole MG, Primeau F, Bellavance F. Delirium risk factors in elderly hospitalized patients. J Gen Intern Med. 1998;13:204-12.

56. Inouye Sk, Bogardus ST, Charpentier PA et al. A multicomponent intervention to prevent delirium in hospitalized older patients. N Engl J Med.1999; 340: 669-76

57. Furlaneto Maria Elizabet, Garcez-Leme Luiz Eugênio. Delirium in elderly individuals with hip fracture: causes, incidence, prevalence, and risk factors. Clinics [serial on the Internet]. 2006 Feb [cited 2011 May 24] ; 61(1): 35-40. Available from: http://www.scielo.br/scielo.php?script=sci_arttext\&pid=S180759322006000100007\&lng=en. doi: 10.1590/S1807-59322006000100007

58. Veiga Fernández F, Cruz Jentoft AJ. Etiología y fisiopatología del delirium. Re Esp Geriatr Gerontol.2008;43(Supl 3):4-12.

59. Gayoso Orol MJ, Sanz Casalengua AM. Cuadros confusinales agudos. In: Guillen Llera F, Pérez del Molino Martín J. Petidier Torregrossa R, editores. Sindromes y cuidados en pacientes geriátricos. Protocolos de actuación.2ª ed., Barcelona: Elsevier Masson; 2008. p.547-6.

60. Lipowski ZI. Delirium: acute confusional states. New York: Oxford University Press, 1990.

61. Schor JD, Levkoff SE, Lipsitz LA, Reilly CH, Cleary PD, Rowe JW et al. Risk factors for delirium in hospitalized elderly. JAMA. 1992;267:827-31.

62. Juliebø V, Bjøro K, Krogseth M, Skovlund E, Ranhoff AH, Wyller TB. Risk. factors for preoperative and postoperative delirium in elderly patients with hip fracture. J Am Geriatr Soc. 2009;57(8):1354-61.

63. Francis J. Delirium. In Cassel CK (ed.). Geriatric medicine. 3rd ed. New York; Springer, 1997. p. 917-22.

64. Dobmeyer K. Delirium in elderly medical patients. Clin Geriatr. 1996; 4: 43-68.

65. Carnes M. Diagnosis and management of delirium in the elderly. Clin Geriatr. 1996; 4: 16-32.

66. Bross MH, Tatum NO. Delirium in the elderly patient. Am Fam Physician. 1994; 50: 132532.

67. Rummans TA, Evans JM, Krahn LE, Fleming KC. Delirium in elderly patients: evaluation and management. Mayo Clin Proc. 1995; 70: 989-98.

68. Ballabriga Planas J, Martínez Yélamos S, Martínez Yélamos A, Arbizu Urdiain T. Delirios y cuadros confusionales. Etiología, clasificación y diagnóstico. Medicine. 1998;7(93):4342-47. 
69. Duppils GS, WikBlad K. Delirium: behavioural changes before and during the prodromal phase. J Clin Nurs. 2004:13;609-16

70. Lipowski ZJ.Delirium: How its concept has developed. Int Psychogeriatr. 1991;3(2):11520

71. Levkoff SE, Safran C, Cleary PD. Identification of factors associated with the diagnosis of delirium in hospitalized elderly patients. J Am Geriatr Soc. 1988;36:1099-104.

72. O'Keefe ST, Lavan JN. Clinical significance of delirium subtypes in older people. Age Ageing. 1999;28:115-9.

73. Winawer N. Postoperative delirium. Med Clin North Am. 2001;85:1229-39.

74. Siddiqi N, House AO, Holmes JD. Occurrence and outcome of delirium in medical in patients: a systematic literature review. Age Ageing. 2006;35: 350-64.

75. Chan D, Brennan NJ. Delirium: making the diagnosis, improving the prognosis. Geriatrics .1999;54:28-42.

76. Shapira J, Roper J, Schuzinger J. Pacientes con delirio. Nursing Español .1994;12:4952.

77. Inouye SK, Schlesinger MJ, Lydon TJ. Delirium: a symptom of how hospital care is failing older persons and a window to improve quality of hospital care. Am $\mathrm{J}$ Med. 1999;106:565-73.

78. Marcantonio ER, Flacker JM, Wright RJ, Resnick NM. Reducing delirium after hip fracture: a randomized trial. J Am Geriatr Soc. 2001;49:516-22.

79. Gustafson $Y$, Brannstrom B, Berggren D, et al. A geriatric-anesthesiologic program to reduce acute confusional states in elderly patients treated for femoral neck fractures. $J$ Am Geriatr Soc. 1991;39:655-62.

80. Rockwood K, Cosway S, Stolee P, Kydd D, Carver D, Jarret P, O'Brien B. Increasing the recognition of delirium in elderly patients. J Am Geriatr Soc. 1994;42:252-6.

81. Lewis LM, Miller DK, Morley JE, Nork MJ, Lasater LC. Unrecognized delirium in ED geriatric patients. Am J Emerg Med. 1995;13(2):142-5.

82. Pompei $P$, Foremam M, Rudberg MA, Inouye SK, Braund V, Cassel CK. Delirium in hospitalized older persons: outcomes and predictors. J Am Geriatr Soc. 1994;42:809-15.

83. Rincón HG, Granados M, Unutzer J, Gómez M, Durán R, Badiel M, et al. Prevalence, detection and treatment of anxiety, depression and delirium in the adult critical care unit. Psychosomatics. 2001;42(5):391- 6.

84. Elie M, Rousseau F, Cole M, Primeau F, McCusker J, Bellavance F. Prevalence and detection of delirium in elderly emergency department patients. CMAJ. 2000;163(8):97781.

85. Milisen K, Foreman MD, Wouters B, Driesen R, Godderis J, Abraham IL, et al. Documentation of delirium in elderly patients with hip fracture. J Gerontol Nurs. 2002 ;28(11):23-9.

86. Inouye SK. The dilemma of delirium: clinical and research controversies regarding diagnosis and evaluation of delirium in hospitalized elderly medical patients. Am J Med. 1994; 97:278-88.

87. Inouye SK. A practical program for preventing delirium in hospitalized elderly patients. Cleveland Clin J Med. 2004; 71: 890-6.

88. Álvarez-Fernández B, Gómez-Huelgas R. Síndrome confusional agudo en el paciente hospitalizado. En: López Soto A, Formiga Pérez F, Ruiz Hidalgo D, Duaso Magaña E, editores. Clínicas en geriatría hospitalaria. Barcelona; 2006. p. 29-42.

89. Ruiz. D. Pronostico del síndrome confusional agudo en ancianos. Rev Mult Ger. 2007;17:685-9.

90. Levkoff SE, Evans DA, Liptzin B, Cleary PD, Lipsitz LA, Wetle TT, et al. Delirium. The occurrence and persistence of symptoms among elderly hospitalized patients. Arch Intern Med. 1992;152:334-40. 
91. Brown TM, Boyle MF. ABC of psychological medicine. Delirium. BMJ. 2002; 325: 644647.

92. Registered Nurses Association of Ontario (RNAO). Screening for Delirium, Dementia and Depression in Older Adults. Ontario: Nursing Best Practice Guidelines Project; 2003.

93. González M, Carrasco M. Delirium: marcador del estado del paciente geriátrico. Rev Esp Geriatr Gerontol. 2008;43(Supl 3):38-41.

94. Cole MG, Primeau FJ. Prognosis of delirium in elderly hospital patients. Can Med Assoc J. 1993;149:41-6.

95. Kelly K, Zisselman M, Cutillo-Schmitter T, Reichard R. Gravedad y cursodel delirium en pacientes institucionalizados y hospitalizados por problemas médicos. Am J Geriatr Psychiatry. 2001;2:267-73.

96. González M, Martínez G, Caldero'n J, Villarroel L, Yuri F, Rojas C, et al. Impact of Delirium on Short-Term Mortality in Elderly Inpatients: A Prospective Cohort Study. Psychosomatics. 2009;50:234-8

97. Marcantonio ER, Kiely DK, Simon SE, John Orav E, Jones RN, Murphy KM, Bergmann MA: Outcomes of older people admitted to postacute facilities with delirium. J Am Geriatr Soc.2005. $53:$ 963-69.

98. Schofield I. Delirium: challenges for clinical governance. J Nurs Manag. 2008;16:127-33.

99. Dolan MM, Hawkes WG, Zimmerman SI, Morrison RS, Gruber-Baldini AL, Hebel JR, et al. Delirium on hospital admission in aged hip fracture patients: prediction of mortality and 2-year functional outcomes. J Gerontol A Biol Sci Med Sci. 2000;55:M527-34.

100. Rogers AC, Gibson $\mathrm{CH}$. Experiences of orthopaedic nurses caring for elderly patients with acute confusion. Journal of Orthopaedic Nursing. 2002;6:9-17

101. Lazarus HR, Hagens $\mathrm{JH}$. Prevention of psychosis following open-heart surgery. Am J Psychiatny. 1968; 124: 1190-5.

102. Budd S, Brown W. Effect of a reorientation technique on postcardiotomy delirium. Nurs Res. 1974;23(4):341-8.

103. Chatham MA. The effect of family involvement on patients manifestations of postcardiotomy psychosis. Heart \& Lung: The Journal of Critical Care.1978;7(6):995-9.

104. Owens JF, Hutelmyer CM. The effect of postoperative intervention on delirium in cardiac surgical patients. Nurs Res. 1982;31(1):60-2.

105. Wanich CK, Sullivan-Marx EM, Gottlieb GL, et al. Functional status outcomes of a nursing intervention in hospitalized elderly. Image J Nurs Sch. 1992;24(3):201-7.

106. Tabet N, Hudson S, Sweeney V, et al. An educational intervention can prevent delirium on acute medical wards. Age Ageing. 2005;34(2):152-56

107. Lundström M, Edlund A, Karlsson S, Brännström B, Bucht G, Gustafson Y. A multifactorial intervention program reduces the duration of delirium, length of hospitalization, and mortality in delirious patients. J Am Geriatr Soc. 2005;53(4):622-8.

108. Gentric A, Le Deun $P$, Estivin S. Prevention of delirium in an acute geriatric care unit. Rev Med Interne. 2007;28(9):589-93.

109. Vidan MT, Sánchez E, Alonso M, Montero B, Ortíz J,Serra JA. An intervention integrated in to daily clinical practice reduces the incidence of delirium during hospitalization in elderly patients. J Am Geriatr Soc.2009;57(11):2029-36.

110. Williamns MA, Campbell EB, Raynor WJ, et al. Reducing acute confusional states in elderly patients with hip fractures. Res Nurs Health. 1985;8(4):329-37.

111. Strömberg L, Ohlén G, Nordin C, Lindgren U, Svensson O. Postoperative mental impairment in hip fracture patients. A randomized study of reorientation measures in 223 patients. Acta Orthop Scand. 1999;70(3):250-5.

112. Lundström M, Edlund A, Lundström G, Gustafson Y. Reorganization of nursing and medical care to reduce the incidence of postoperative delirium and improve rehabilitation outcome in elderly patients treated for femoral neck fractures. Scand J Caring Sci. 1999; 13(3):193-200. 
113. Björkelund KB, Hommel A, Thorngren KG, Gustafson L, Larsson S, Lundberg D. Reducing delirium in elderly patients with hip fracture: a multi-factorial intervention study. Acta Anaesthesiol Scand. 2010;54(6):678-88.

114. Nagley SJ. Predicting and preventing confusion in your patients. J Gerontol Nurs 1986;12(3):27-31.

115. Siddiqi N, Stockdale R, Britton AM, Holmes J. Intervenciones para la prevención del delirio en pacientes hospitalizados (Revisión Cochrane traducida). En: La Biblioteca Cochrane Plus, 2008 Número 4. Oxford: Update Software Ltd. Disponible en: http://www.update-software.com. (Traducida de The Cochrane Library, 2008 Issue 3. Chichester, UK: John Wiley \& Sons, Ltd.).

116. Chrispal A, Mathews KP, Surekha V. The clinical profile and association of delirium in geriatric patients with hip fractures in a tertiary care hospital in India. J Assoc Physicians India. 2010;58:15-9.

117. Sieber FE. Postoperative delirium in the elderly surgical patient. Anesthesiol.Clin. 2009;27(3):451-64.

118. Gaspar Alonso-Vega G, Lázaro Marí MP. Tratamiento del síndrome confusional agudo. JANO.2002; 62(1431):33-37.

119. Barrero Raya MC, Parras García de León N. Delirium en el anciano. Salud Rural 2001; XVIII (12): 11-49.

120. Duaso E, Formiga F, Lopez-Soto A, Mañas M, Gómez N. Prevención y tratamiento del delirium. Rev Mut Ger.2007;17:83-8.

121. González Pérez Augusto César, Llibre Rodríguez Juan de Jesús. Acute confusional state in the aged patient. Rev Cub Med Mil [revista en la Internet]. [citado 2011 Jul 15]. Disponible en: http://scielo.sld.cu/scielo.php?script=sci_arttext\&pid=S0138$65572001000500014 \& \operatorname{lng}=\mathrm{es}$.

122. Rubin FH, Neal K, Fenlon K, Hassan S, Inouye SK. Sustaibility and scalability of the hospital elder life program at a community hospital.JAM Geriatr Soc. 2011:59;359-65.

ISSN 1695-6141

๑ COPYRIGHT Servicio de Publicaciones - Universidad de Murcia 